\title{
Paraconsistent Artificial Neural Network Applied in Breast Cancer Diagnosis Support
}

\author{
Carlos Arruda Baltazar ${ }^{2}$, Fábio Vieira do Amaral ${ }^{1}$, Jair Minoro Abe ${ }^{1}$, Alexandre Jacob \\ Sandor Cadim ${ }^{2}$, Caique Zaneti Kirilo ${ }^{2}$, Fábio Luís Pereira ${ }^{1}$, Hélio Côrrea de Araújo ${ }^{1}$, \\ Henry Costa Ungaro ${ }^{2}$, Lauro Henrique de Castro Tomiatti ${ }^{2}$, Luiz Carlos Machi \\ Lozano $^{2}$, Renan dos Santos Tampellini ${ }^{2}$, Renato Hildebrando Parreira ${ }^{1}$, Uanderson \\ Celestino $^{1}$ \\ ${ }^{1}$ Paulista University-UNIP, Graduate Program in Production Engineering, Dr. Bacelar 1212, \\ CEP 04026-002 São Paulo - SP, Brazil \\ favamaral@gmail.com, jairabe@uol.com.br, f.luis01@gmail.com, \\ hhca@globo.com, renatodoryusen@hotmail.com.br, \\ uandersoncelestino@hotmail.com \\ ${ }^{2}$ Paulista University-UNIP, Ungraduate Computer Science, St. Vergueiro 1211, CEP \\ 01504-000 São Paulo - SP, Brazil \\ carlosarrudabaltazar@gmail.com, cardimajs@gmail.com, \\ caiquez.kirilo@hotmail.com, henry_tnt_07@hotmail.com, \\ tomiatti1@hotmail.com, luizlozano@icloud.com, \\ renan.tampellini@hotmail.com
}

\begin{abstract}
In this work, a Paraconsistent Classifier for the diagnosis of breast cancer based on the attributes of mamographic images was developed. The system uses a neural network with decision making from the final results of processing each set of attributes was created. In order to mitigate the effects of false positives diagnoses and true positives. In order to analyze the performance of the Paraconsistent classifier, the results will be compared with the results of the following classifiers: Multi-Layer Perceptron (MLP), a dual stage classifier (ART2LDA) based on Adaptive Resonance Theory (ART) and a classifier implemented with nonlinear optimization techniques and combinatorics, associated with the classification capabilities of Radial basis Functions - (RBF-Simulated Annealing).To perform the simulations, two different databases were used. The first one, to classify calcifications, is composed of 143 samples divided into 64 benign cases and 79 malignant cases represented by form. The performances of the classifiers in discriminating benign and malignant cases are compared in terms of area under the Receiver Operating Characteristic Curve (Az). The higher the value of $\mathrm{Az}$, the better the performance of the classifier.The
\end{abstract}


experiments with calcifications show: Paraconsistent Classifier $(\mathrm{Az}=0.986)$, MLP classifier $(\mathrm{Az}=0.70)$, ART2LDA Classifier $(\mathrm{Az}=0.696)$ and RBF Classifier Simulated Annealing $(\mathrm{Az}=0.94)$. For experiments with mammographic masses and tumors show: Set 1, Paraconsistent Classifier $(\mathrm{Az}=0.939)$, MLP classifier $(\mathrm{Az}=$ 0.994), ART2LDA Classifier $(\mathrm{Az}=0.901)$ and RBF Classifier - Simulated Annealing $(\mathrm{Az}=0.912)$. Set 2, Paraconsistent Classifier $(\mathrm{Az}=0.935)$, MLP classifier $(\mathrm{Az}=$ 0.994), ART2LDA Classifier $(\mathrm{Az}=0.890)$ and RBF Classifier - Simulated Annealing $(\mathrm{Az}=0.924)$. Set 3, Paraconsistent Classifier $(\mathrm{Az}=0.875)$, MLP classifier $(\mathrm{Az}=$ 0.970), ART2LDA Classifier $(\mathrm{Az}=0.850)$ and RBF Classifier - Simulated Annealing $(\mathrm{Az}=0.996)$. Set 4 , Paraconsistent Classifier $(\mathrm{Az}=0.500)$, MLP classifier $(\mathrm{Az}=$ 0.887), ART2LDA Classifier $(\mathrm{Az}=0.767)$ and RBF Classifier - Simulated Annealing $(\mathrm{Az}=0.907)$. Set 5, Paraconsistent Classifier $(\mathrm{Az}=0.929)$, MLP classifier $(\mathrm{Az}=$ 0.987), ART2LDA Classifier $(\mathrm{Az}=0.884)$ and RBF Classifier - Simulated Annealing $(\mathrm{Az}=0.998)$. Set 6 , Paraconsistent Classifier $(\mathrm{Az}=0.939)$, MLP classifier $(\mathrm{Az}=$ 0.982), ART2LDA Classifier $(\mathrm{Az}=0.885)$ and RBF Classifier - Simulated Annealing $(\mathrm{Az}=0.999)$. In the case of the Paraconsistent Classifier, the eighth experiment was composed of the total image attributes relating to mammographic masses and tumors $(\mathrm{Az}=0.939)$. In the particular case of the RBF-Sort Simulated Annealing experiment with all the image attributes, it was proved its unfeasibility, due to the complexity of their algorithm, where the processing time tends to infinity for a larger number of elements. For the experiments, the Paraconsistent classifier used 20\% of the samples for the neural network training, against the total number of samples available minus one for the other classifiers.

Keywords: Attributes of Images Classifier, Breast cancer, Computer-Aided Diagnosis, Paraconsistent Annotated Evidential Logic Et, Paraconsitent Artificial Neural Network Mammography

\section{INTRODUCTION}

Breast cancer fills second place in cancer occurrences in the world, with $22 \%$ of all the new cases each year. If diagnosis point and treatment occurs in start, healing prognosis is favorable. [3]

In world population, the average after life reach five years after diagnosis in $61 \%$ of the cases.

Brazil epidemiological illness profile subdued special attention in politic decisions of federal government. Breast cancer brings high mortality rate, because is only diagnosed in final stages. The Plan for Strengthening Prevention Actions and Qualification of the Diagnosis and Treatment of Cervical and Breast Cancers, released on March 2012, is an example of this premise. The incentive of X-ray exam realization 
in breast of caring population also belongs in this prevention politics. [1][2]

Breast X-ray (mammography) is the most indicated for detect early the presence of nodes inside breasts. Clinical exam and others image and lab exams could also support the diagnosis.

The exam shows a variety of adipose tissue, parenchyma and normal and abnormal tissues. The radiologist should analyze abnormally, using attributes, dimension, form and texture. After analysis is possible to identify illness presence. Then analyze if the node is actually a bad one, besides the fact that most of them are not. [1][2]

The risk of a wrong diagnosis, false positive or false negative, is high. Clinical exams shows that some Brazilian cities has $50 \%$ of mammography tests with difficult in reading and diagnosis. The process realized during the exam is highly important for exams quality. [1][2]

For reach acceptable trust level, is necessary to raise the prediction of positive values. The percentage of breast cancer cases identified in mammography and confirmed by biopsy that can reach $30 \%$. Considering the traumatic aspect and costs for biopsy realization, it is relevant an alternate computational capable of raise distinguish between good and bad items found. This method could work well as decision-making tool. The radiologist would use this tool as part of the analysis protocol of mammography. With this, the wrong diagnosis would been minimized and the exam recommendations of biopsy reduced. [1][2]

This work treat to propose a tool capable of analyze and classify good and bad mammography attributes and a change in the process.

Attribute image classification will be supported by paraconsistent artificial neural network. This tool does not manipulate mammography. In this part of the work will be treated only aspects related to calcification. To practice this simulations will be used a database, obtained from segmentations treated in mammography acquired with Department of Radiological Sciences and Diagnosis Imaging Foothills Hospital, located in Calgary, Alberta, Canada, this database is built from 69 good cases and 79 bad ones, identified manually from a radiologist specialized in mammography. From this point, the identifications determine the factors in Momentum (m), Compaction factor (c) and Fourier description (f). [1][2]. 


\section{Digital IMAges AtTributes}

The computed detection of good masses, bad tumors and calcification in mammography are not simply filled with Neural Networks specialized in ordinary recognition. In existing ordinary recognition (as mammography) built by neural networks, identify forms from borders and patterns (image segmentation), so, localizing frontiers between background and pattern. In this recognizing, the better image definition of frontier in patterns the better results of border detection. In mammography, most of the good masses, just like good calcifications, has well-defined aspects and frontier (borders) with high contrasted degree. Forms of good calcifications can be circular and oval shaped. Contour of the benign masses beyond the circular and oval form, could also present the macro lobular form, shown in picture 2. The border transition is clear from inside the dense tissue from outside, less dense. Malignant calcifications, although, seems to have cloudy contrast and frontier not so clear. Malignant calcification outlines shows lobular shape, and malignant tumors shows microlobulated shape. In this case, the transition of interne tissue (denser), from extern is not very clear or defined. However, some benign masses such as fiber adenoma could present erased borders in mammography.

About the convexity and concavity of masses shape, we could see that the most benign masses presents convex shapes. Some of them are concaved or speculated. However, malignant tumors could present both convex and concaved, and even predominant tentacles shape. And if compared, benign masses and malignant tumors, are both homogeneous in terms of density and distribution.

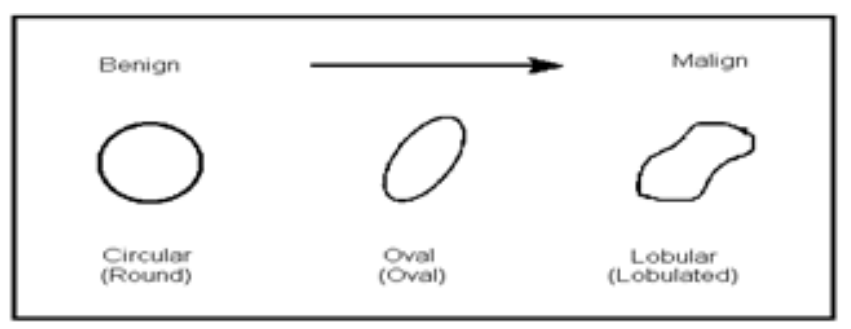

Fig. 1 - Morphology of calcification visualized in Mammography. 


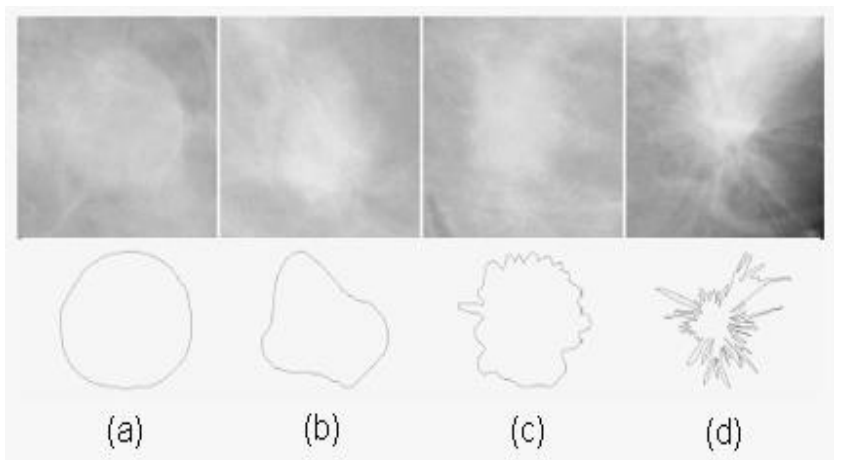

Fig. 2 - Shape examples, benign (a) and (b), malign (c) and (d).

Given these facts, if a neural network was trained for pattern recognizing of masses and benign and malign calcification, it would be, depending on network implementation, an efficient benign masses recognizer, but not so efficient detecting malign tumors, sometimes not recognizing fiber adenomas. Therefore, neural networks trained to recognize only patterns are not well suited and recommended to support cancer diagnosis, because it lacks where radiologists need it the most, identify cancer.

The fact that the simply pattern recognizing has few or no application in mammography, shape factors, distinctness detection of masses outline and measures of texture are the answer CAD (Computer-Aided Diagnosis) for diagnosing attributes extraction in mammography.

Parameters that describe calcifications identified in mammography:

- $\quad$ Momentum (m)

- $\quad$ Fourier (f)

- $\quad$ Compaction factor (C)

- $\quad$ Radial (r)

- $\quad$ Distance (d)

- Standard detour of the radial distance

- $\quad$ Tumor Border Roughness Index(R)

- $\quad$ Índice de forma espiculada (SI): 
- $\quad$ Entropia:

- $\quad$ Segundo momento:

- $\quad$ Diferença de momento:

- Inverso da diferença do momento:

- Correlação: medida da dependência linear do brilho:

- $\quad$ Ac (acutance)

\section{PARACONSISTENT ARTIFICIAL NEURAL NETWORK}

Features of connectionist structure composed of an extensive network components supported by paraconsistent Annotated Evidential Et. [7][8]

These Artificial Neural Networks are built from Artificial Neural Cells forming Paraconsistent Artificial Neural Units Paraconsistent (UNAP's) and Arificial Paraconsistent Neural Systems (SNAP's) that direct, treat and analyze information signals obtaining a close model of the mental functions that characterize the behavior of the human brain. [7][8]

Based on these criteria and foundations that enable the implementation of CNAP's necessary recognition system were established. [7][8]

- Analytical Connection

- Simple Logic Connecton

- Selective Logic Connection

- Passageway

- Complementation

- Decision

- Learning

- Memorization

\section{A. Artificial Paraconsistent Learning Cell (CNAPa)}

As part of this memory unit or as standards in primary sensor layers, in other words, all information learned or stored, must pass for such cell. 


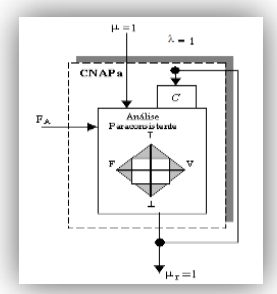

Picture 4 - Representation of CNAPa

\section{B. Artificial Paraconsistent Decision Cell (CNAPd)}

This cell act as a node of decision in RNAP's. In your entry, applying two signals from another network area, resulting in only one exit that matches one conclusive analysis of these entries.

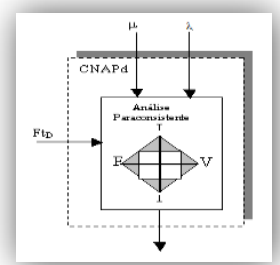

Picture 5 - Representation of CNAPd [7][8]

The possible output values are:

- Output equals to 1 represents the pattern "True".

- Output equals to 0 represents the pattern "Falsity".

- Output equals to 0.5 represents the pattern "Uncertainty". 


\section{Simple Logic Artificial Neural Paraconsistent Cell (CNAPCls)}

Establishes the logical connective OR (maximization) between degrees of belief applied to the input of the cell, telling you which of the two signals applied to the input of the cell has the highest value, setting the signal at the output.

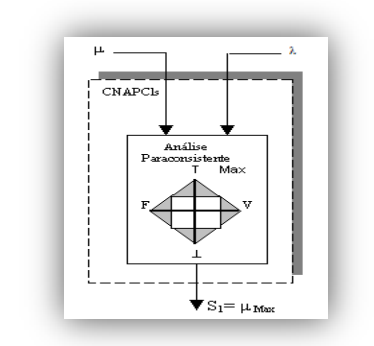

Picture 6 - Representação de uma CNAPCls [7][8]

\section{Paraconsistent Analyzer}

Considering traumatic aspects and the cost for realization of biopsy, and is relevant an alternative computational way capable of raise distinguish between benign and malign calcification. This method works like a decision support tool. The radiologist would use this tool as a protocol stage, helping mammography analysis. With this, wrong diagnosis would been lowered and biopsy exam recommendation.

This software will implement a RNAP for each image attribute ( $\mathrm{m}, \mathrm{c}$ and $\mathrm{f}$ ), and in the process ending it will show the diagnosis.

In the image below, the process of paraconsistent classification. This process will be similar to calcification classifications. 


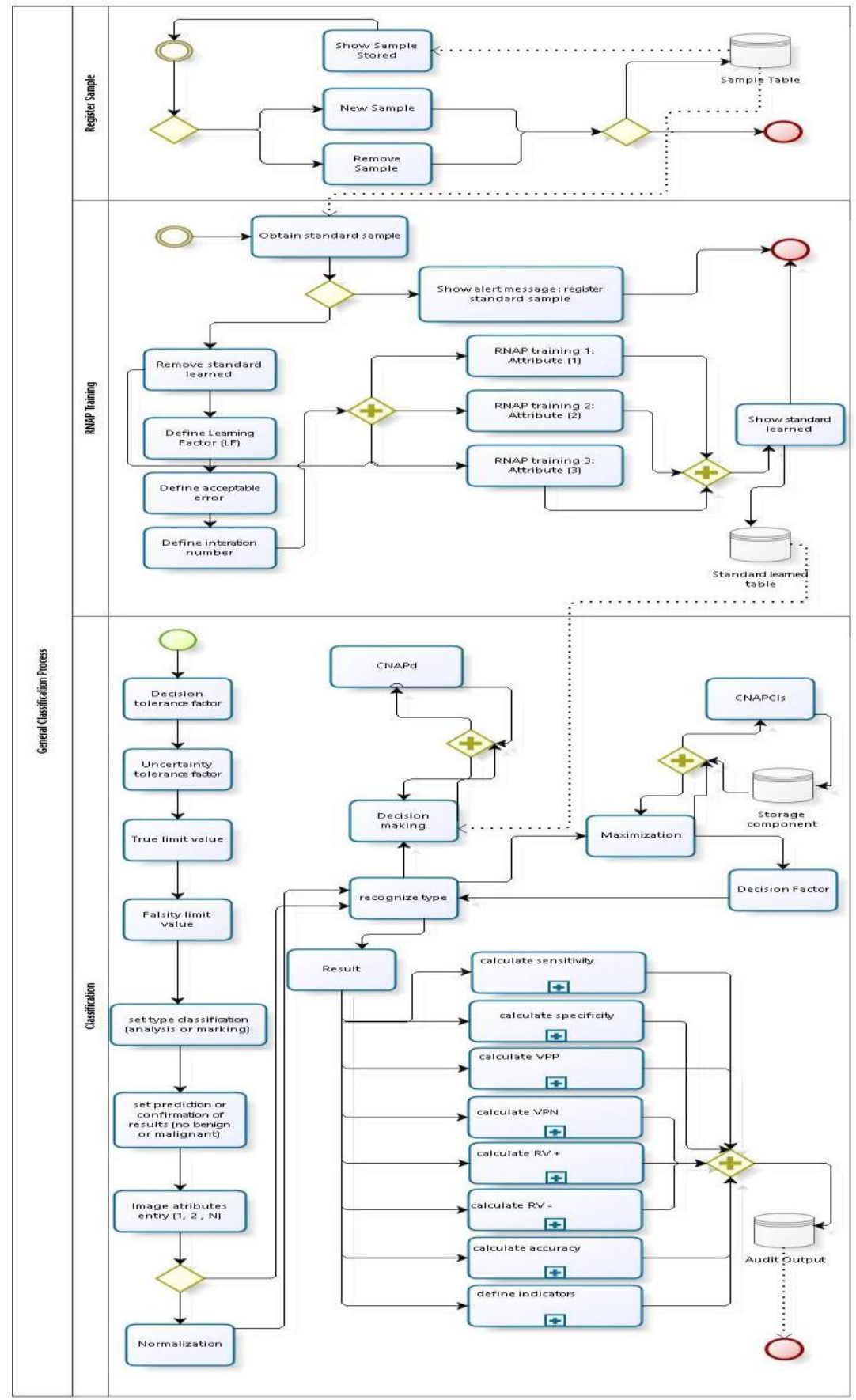




\section{A. Network composition}

Main part of the network is built by a group of CNAPa that learn and spread for others parts of the network.

The second layer is composed by CNAPd, aiming stabilish the evidence degree between standard stored in CNAPa.

The third layer is built by CNAPCI, created for maximization, forming a competitive network, in which only one value is understood as winner.

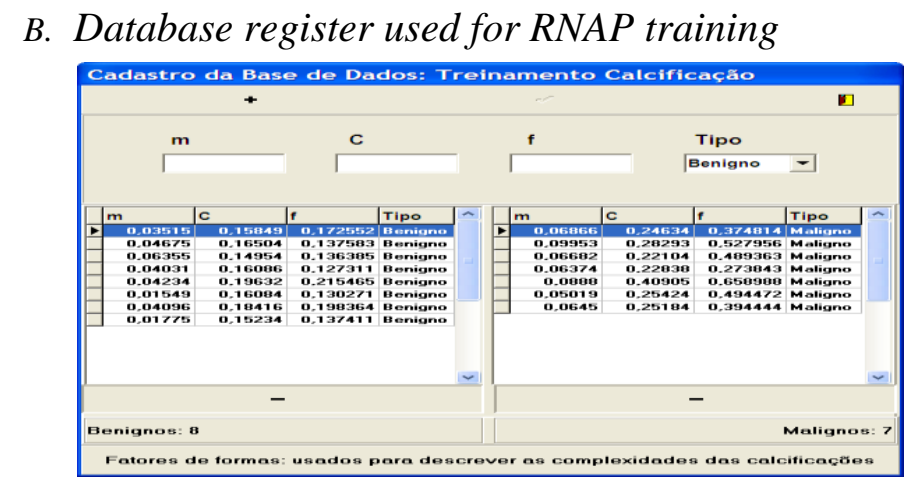

For network training it is not necessary a high number of values.

C. RNAP training

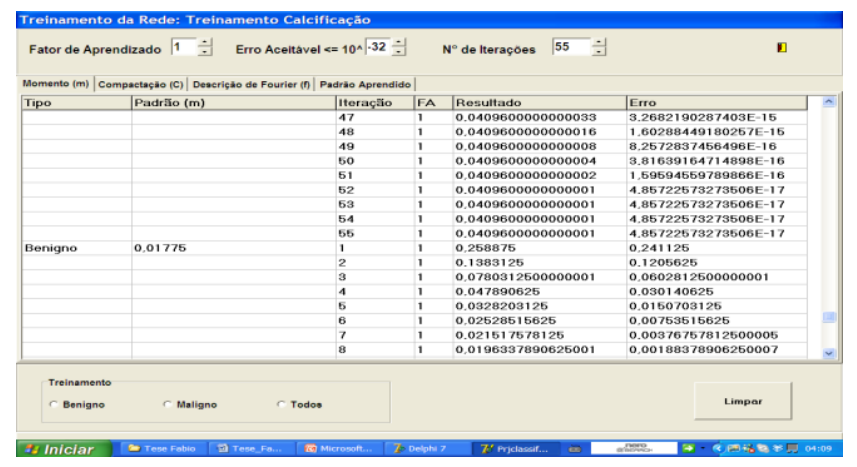




\section{RNAP classification}

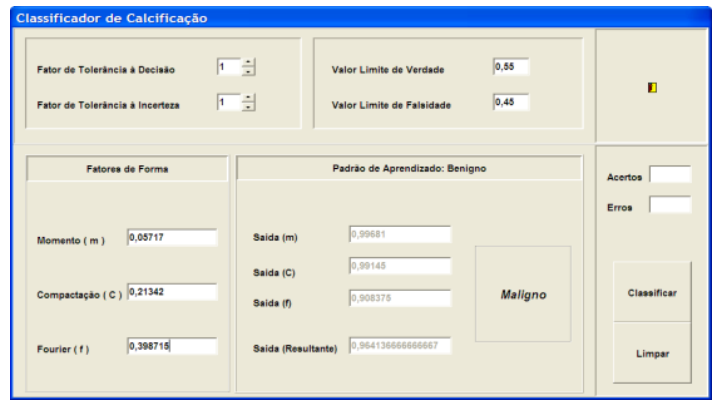

This module function can obtain a value resulting of network process, capable of determinate if a newer sample is benign or malign, and shows quality and reliability of classification through calculations of sensibility, specificity, predicted positive value, predicted negative value, likelihood positive reason, likelihood negative reason and accuracy. And how much true positive, false positive, true negative and false negative it has.

\section{v. Final Conclusions}

The paraconsistent analyzer in its first version could reach $94 \%$ hits for calcification.

Considering the diagnosis errors could reach $50 \%$ in extreme cases, there was a significant reduction of diagnosis with false positives or false negatives.

RNAP characteristics also need to be quoted.

- Low number of samples needing.

- In this tool case, there was no detraining in network, so required less time training.

- The algorithm complexity implemented also presents easy mastery.

\section{REFERENCES}

[1] Espírito-Santo, Rafael. Discriminação de Classes Fundamentada em Técnicas de Otimização Não Linear e Combinatória Associadas a Funções de Base Radiais: Um estudo de Caso em Mamografia. Tese apresentada à Escola Politécnica da Universidade de São Paulo para o obtenção do título de Doutor em Engenharia. Área de Concentração: Sistemas Eletrônicos. São Paulo, 2004. 
[2] Espírito, S.Rafael; Lopes, Roseli de Deus; Rangayyan, Rangaraj M. Classification of Breast Masses in Mammograms Using Radial Basis Functions and Simulated Annealing. International Journal of Cognitive Informatics and Natural Intelligence, 3(3),27-38, JulySeptember 2009. Vol. 3, No. 3. www.igi-global.com

[3] Instituto Nacional de Câncer José Alencar Gomes da Silva. Coordenação Geral de Ações Estratégicas. Coordenação de Prevenção e Vigilância. Estimativa 2012 : incidência de câncer no Brasil / Instituto Nacional de Câncer José Alencar Gomes da Silva, Coordenação Geral de Ações Estratégicas, Coordenação de Prevenção e Vigilância. - Rio de Janeiro : Inca, 2011.

[4] Da Costa,N.C.A.; Abe, J.,M.; Murolo, A. C.; Da Silva Filho, J. I.; Leite, C. F. S. Lógica Paraconsistente Aplicada. São Paulo, Atlas, 1999

[5] Da Silva Filho, J. I.; Abe, J. M. Fundamentos das Redes Neurais Artificiais Paraconsistentes destacando aplicações em Neurocomputação. São Paulo, Arte \& Ciência, p.298, 2001.

[6] Abe, J. M.; Da Silva Filho, J. I.; Celestino, U.; Corrêa, de A. H.. Lógica Paraconsistente Anotada Evidencial Et. Santos, Editora Comunicar, 2011.

[7] Pereira, Fábio Luís. Reconhecimento de Caracteres Numéricos Manuscritos Utilizando Redes Neurais Artificiais Paraconsistentes. Dissertação apresentada ao Programa de PósGraduação em Engenharia de Produção da Universidade Paulista para obtenção do título de Mestre em Engenharia de Produção. São Paulo, 2011.

[8] Silva, F.J.I.; Abe, J.M. Fundamentos das Redes Neurais Artificiais Paraconsistentes. São Paulo. Arte\&Ciência, 1999. 